\title{
CD59 receptor targeted delivery of miRNA-1284 and Cisplatin-loaded liposomes for effective therapeutic efficacy against cervical cancers
}

\section{Li Wang}

Jining No 1 People's Hospital

Ting-Ting Liang ( $\sim$ TrevonBraunrba@yahoo.com )

Weifang No. 2People's Hospital

\section{Research article}

Keywords: Cervical cancers, liposomes, miRNA-1284, cisplatin, apoptosis

Posted Date: September 19th, 2019

DOI: https://doi.org/10.21203/rs.2.14621/v1

License: (c) (i) This work is licensed under a Creative Commons Attribution 4.0 International License.

Read Full License

Version of Record: A version of this preprint was published at AMB Express on March 17th, 2020. See the published version at https://doi.org/10.1186/s13568-020-00990-z. 


\section{Abstract}

Background: In this study, we have designed the CD59sp-conjugated miRNA-1284/cisplatin(CDDP)loaded liposomes for the enhanced therapeutic effect against cervical cancers. Methods: A substantially higher uptake of CD59 antibody-conjugated miRNA-1284/CDDP-loaded liposomes (CD/LP-miCDDP) was attributed to the CD59-based receptor-mediated cellular internalization in HeLa cells. Results: MiR-1284 showed a typical concentration-dependent cell killing effect in the cervical cancer cells owing to the downregulation of HMGB1. CD59-antibody conjugated formulation exhibited a significantly lower cell viability compared to that of free CDDP and non-targeted LP-miCDDP. The combination of miR-1284 and CDDP could result in a synergistic anticancer effect on the cervical cancer cells. The IC50 value of CDDP, LP-miCDDP and CD/LP-miCDDP was observed to be $12.4 \mu \mathrm{g} / \mathrm{ml}, 7.23 \mu \mathrm{g} / \mathrm{ml}$ and $3.12 \mu \mathrm{g} / \mathrm{ml}$, respectively. CD/LP-miCDDP exhibited remarkable cell/nuclei rupturing and apoptotic bodies' formation indicating a severe apoptosis effect in HeLa cancer cells. Flow cytometer analysis showed that CD/LP-miCDDP resulted in maximum apoptosis effect ( $60 \%)$ compared to CDDP ( 20\%) or miR-1284 ( 12\%) treated cells indicating the superior anticancer effect in the cancer cells. Conclusions: Finally, CD/LP-miCDDP significantly prolonged the blood circulation of encapsulated drug in rats with significantly higher AUC (ot) , $t 1 / 2$ and lower $C L$ compared to that of free CDDP. Overall, CD/LP-miCDDP could hold a promising potential in the treatment of cervical cancers.

\section{Background}

Cervical cancer is one of the major world problems and considered to be one of common gynecologic cancers making it as fourth most cancer related death among women [1]. Approximately, $85 \%$ of cervical cancer cases originate from the developing countries such as China. According to one statistic, 87,982 people were diagnosed with cervical cancer in 2011 in China and more than 20,000 patients have been died in the same year [2,3]. The global incidence of cervical cancer kept increasing with more than 250,000 deaths each year. Based on the guidelines of NCCN for cervical cancer, cisplatin (CDDP) is considered as a first line of treatment option [3]. CDDP alone or in combination with paclitaxel has been recommended for advanced or metastatic cancers. At times, treatment includes radiotherapy or surgery; however it met with severe complications such as non-specificity and aggressiveness [4]. Besides, chemotherapy with CDDP or combination with other chemotherapeutic agents results in severe doselimiting toxicity such as nephrotoxicity or hepatotoxicity and leads to severe drug resistance [5].

Therefore, potential therapeutic approaches by which drug toxicity could be reduced and chemosensitivity of CDDP towards cervical cancer could be increase is perused continuously.

MicroRNAs (miR) are typically consists of a chain of 17-25 nucleotides non-coding short RNAs and binds to the specific mRNAs at the $3^{\prime} U T R s$ [6]. Several of the miRNAs are indicated as key regulators in the proliferation, differentiation and regulation of cancer cell growth by acting on the gene expression at post-transcriptional level [7]. Studies have demonstrated that miRNAs can exhibit the control over cancer cells by controlling the key pathways [8]. In this regard, miR-1284 has been shown to inhibit the cancer cell growth by targeting the downstream targets [9]. Importantly, its expression has been related with the 
chemosensitivity in gastric cancers. Therefore, in this study, we have selected miR-1284 to increase the chemosensitivity of CDDP for the treatment of cervical cancers $[10,11]$.

The free CDDP is known to exhibit severe toxicity to the vital organs due to non-specific distributions and thereby it has limited efficacy in the tumors. Similarly, miRNA is highly unstable in the systemic circulation. Keeping this in mind, nanocarrier-based drug delivery system received great attention owing to potential to improve the physicochemical characteristics of the anticancer drug $[12,13]$. The nanocarriers are demonstrated to enhance the therapeutic efficacy, reduce the unwanted adverse effects, improve the blood circulation, and improve the cellular accumulation in the tumor tissues $[14,15]$. To be specific, liposome has emerged as a one of the most tested carrier system in clinical trials [16]. The unique structure of liposome provides high drug loading, surface modifications, plasma stability and tunable particle size. Although, liposome accumulates in the tumor tissue via enhanced permeation and retention (EPR) effect, targeted carrier remarkably increase the effectiveness of the therapy [16]. Tumor targeted carrier system selectively accumulate in the tumor and kill the cancer cells without causing unwanted side effects. Several receptors are expressed in the cervical cancers such as EGFR, CD44 or HER2 which could be utilized to target the nanoparticles $[18,19]$. Recently, CD59 has been reported to overexpress in the cervical cancers and in other cancers, while is low expressed in normal cells [20]. CD59 is a membrane regulatory component and promote immune escape of tumor cells [21].

Therefore, we have designed unique CD59sp-conjugated CDDP/miR-1284-loaded liposomes for the effective treatment of cervical cancers (Figure 1). The targeting specificity of CD59sp was evaluated by flow cytometry and confocal laser scanning microscopy (CLSM). The in vitro anticancer effect was evaluated by means of cell viability analysis, and apoptosis assay. Finally, pharmacokinetic analysis was performed to determine the blood circulation property of the designed carrier system.

\section{Methods}

\section{Preparation of CD59sp-conjugated CDDP/miRNA-loaded liposomes}

At first, CDDP-loaded liposome was prepared by hydration-sonication method. Briefly, distearoylphosphatidylcholine (DSPC) succinylphosphatidylethanolamine (DSPE-mPEG), distearoyl-N-(3carboxy-propionoylpoly (ethyleneglycol) succinl) phosphatidylethanolamine (DSPE-PEG-COOH), and 1,2dioleoyl-3-trimethyl-ammoniumpropane (DOTAP) were added in a molar ratio of 40:20:10:2 in the chloroform (along with $10 \% \mathrm{w} / \mathrm{w}$ of CDDP). The organic solvent was evaporated in a rotary evaporator at $55^{\circ} \mathrm{C}$ for $2 \mathrm{~h}$. The dried lipid-film was hydrated by incubating in a $1 \mathrm{X}$ PBS for $1 \mathrm{~h}$. The crude dispersion was sonicated for $5 \mathrm{~min}$ using a probe sonicator. To this liposome, $2 \mathrm{mg} \mathrm{EDC}$ was added per $1 \mathrm{mg} / \mathrm{ml}$ of liposome and stirred for $1 \mathrm{~h}$; followed by CD $59 \mathrm{sp}(1 \mathrm{mg} / \mathrm{ml})$ was added to the solution and stirred in dark condition for $4 \mathrm{~h}$. The unconjugated initial ingredients and unconjugated CD59 were removed by centrifugation at $5000 \mathrm{xg}$ for $15 \mathrm{~min}$ at $4^{\circ} \mathrm{C}$. The cationic liposome was then incubated with required amount of miRNA overnight and it is loaded on the surface of liposome. 


\section{Characterization of CD59sp-conjugated CDDP/miRNA-loaded liposomes}

The particle size and polydispersity index (PDI) and zeta potential was determined by dynamic light scattering (DLS) technique using ZetaSizer (Nano series- Nano-ZS ZEN3600, Malvern Instruments, UK). Before the measurement, $0.1 \mathrm{ml}$ of samples was diluted to $1 \mathrm{ml}$ and measured triplicate at $25^{\circ} \mathrm{C}$. The particle morphology was evaluated using transmission electron microscopy (TEM, JEM-2100, JEOL, Japan). The drop of samples were placed in the copper grid and stained with $2 \%$ phosphotungistic acid and dried using infrared light. The particles were then observed under electron microscope.

\section{Drug loading study}

The drug loading and quantification was performed by OPDA-derivatization method. For this purpose, known quantity of dried liposome was added to $100 \mu \mathrm{l}$ of DMF and vortexed. To this, $100 \mu \mathrm{l}$ of OPDA and $200 \mu \mathrm{l}$ of PBS were added and the mixture was heated at $100^{\circ} \mathrm{C}$ for $15 \mathrm{~min}$. The samples were cooled in ice-bath and $1.6 \mathrm{ml}$ of DMF was added to make it to $2 \mathrm{ml}$ as a final volume. Earlier, a calibration curve was performed at different concentration of CDDP and analyzed through UV-Vis spectrophotometer (JASCO-V-730) at a wavelength of $706 \mathrm{~nm}$.

\section{Cellular uptake of CD59sp-conjugated CDDP/miRNA-loaded liposomes}

The cellular uptake study was first performed by confocal laser scanning microscopy (CLSM) method. Briefly, HeLa cells (ATCC, USA) were seeded in 6-well plate with a cover-slip on it and the cells were incubated for $24 \mathrm{~h}$ at $37^{\circ} \mathrm{C}$. Next day, cells were treated with LP-miCDDP and CD/LP-miCDDP formulations, respectively for $2 \mathrm{~h}$. The cells were washed 2 times and immediately incubated with Lysotracker Green ${ }^{\mathrm{TM}}$ for $10 \mathrm{~min}$. The cells were washed and fixed with $4 \%$ paraformaldehyde and stained with DAPI as a nuclear staining. The cover-slip containing cells were mounted on a glass slide and observed under microscope (Leica SP8).

The cellular uptake was further evaluated by flow cytometer. Briefly, HeLa cells were seeded in 6-well plate and the cells were incubated for $24 \mathrm{~h}$ at $37^{\circ} \mathrm{C}$. Next day, cells were treated with LP-miCDDP and CD/LPmiCDDP formulations, respectively for $2 \mathrm{~h}$. The cells were washed and detached with trypsin/ethylenediaminetetraacetic acid mixture and centrifuged a $1200 \mathrm{rpm}$ for 3 mins. The pellet cells were reconstituted with the PBS and studied in BD FACSCalibur flow cytometer (BD FACS, NJ, USA).

\section{In vitro anticancer effect analysis}

The in vitro anticancer effect of free miRNA and the formulations were evaluated by MTT assay. Briefly, briefly, $1 \times 10^{4} \mathrm{HeLa}$ cells were seeded in 96 -well plate and the cells were incubated for $24 \mathrm{~h}$ at $37^{\circ} \mathrm{C}$. The 
cells were either treated with increasing concentration of lipofectamine-miRNA complex or free CDDP, miCDDP and CD/LP-miCDDP formulations or incubated for $24 \mathrm{~h}$. The cells were washed two times with PBS and added with $20 \mu \mathrm{l}$ of MTT solution that has a concentration of $5 \mathrm{mg} / \mathrm{ml}$ and incubated for $4 \mathrm{~h}$ at $37^{\circ} \mathrm{C} .100 \mu \mathrm{l}$ of DMSO was added and incubated for $15 \mathrm{~min}$ and then absorbance was read at $570 \mathrm{~nm}$ using a microplate reader. The untreated cells were taken as a control and IC50 value were calculated from GraphPad Prism software.

\section{Apoptosis analysis-Nuclear staining}

The nuclear morphology of cancer cells after treatment was evaluated by Hoechst 33342 staining. Briefly, $2 \times 10^{5} \mathrm{HeLa}$ cells were seeded in $96-$ well plate and the cells were incubated for $24 \mathrm{~h}$ at $37^{\circ} \mathrm{C}$. The cells were then treated with free CDDP, miCDDP and CD/LP-miCDDP formulations and incubated for $24 \mathrm{~h}$. The cells were washed with PBS twice and fixed with $4 \%$ paraformaldehyde for $10 \mathrm{~min}$. The cells were washed and then stained with $10 \mu \mathrm{g} / \mathrm{ml}$ of Hoechst 33342 solution for $15 \mathrm{~min}$. The cells were washed three times and morphology was observed through IN Cell Analyzer 2000 (GE Healthcare Life Science, USA).

\section{Apoptosis analysis-Flow cytometer}

The quantitative apoptosis was performed by Annexin V-FITC and PI double staining followed by flow cytometer analysis. Briefly, $2 \times 10^{5} \mathrm{HeLa}$ cells were seeded in 96 -well plate and the cells were incubated for $24 \mathrm{~h}$ at $37^{\circ} \mathrm{C}$. The cells were then treated with free CDDP, miCDDP and CD/LP-miCDDP formulations and incubated for $24 \mathrm{~h}$. After $24 \mathrm{~h}$, cells were scrapped gently and centrifuged at $1200 \mathrm{rpm}$ for $3 \mathrm{~min}$. The pellet cells were reconstituted with $100 \mu \mathrm{l}$ of binding buffer and stained with $5 \mu \mathrm{l}$ of Annexin V-FITC and PI and subjected to incubation for $15 \mathrm{~min}$ in the dark atmosphere. The volume was made upto $1 \mathrm{ml}$ and studied using the in BD FACSCalibur flow cytometer (BD FACS, NJ, USA).

\section{Pharmacokinetic analysis}

The Sprague Dawley rats were obtained from In-House Animal Facility, Weifang No.2 People's Hospital, Weifang, China. The experimental animal study protocol was approved by Institutional Animal Ethics Committee of Weifang No.2 People's Hospital, Weifang, China. The pharmacokinetic analysis of free drug and drug-loaded formulations were studied in Sprague Dawley rats $(200+20 \mathrm{~g})$. The rats were given free access to food and water until the study and caged in a standard atmosphere as per the guidelines set by the Institutional committee for animal care and handling. The rats were divided into 3 groups with 5 rats in each group. All the rats received the drug and formulation treatment via the tail vein injection. The CDDP is administered at a dose of $5 \mathrm{mg} / \mathrm{kg}$ while formulations were given $5 \mathrm{mg} / \mathrm{kg}$ equivalent dose. The blood samples were collected from the rats from retro-orbital plexus after $0.25 \mathrm{~h}, 0.5 \mathrm{~h}, 1 \mathrm{~h}, 2 \mathrm{~h} .4 \mathrm{~h}, 6 \mathrm{~h}, 8 \mathrm{~h}$, $12 \mathrm{~h}, 24 \mathrm{~h}, 36 \mathrm{~h}$ and $48 \mathrm{~h}$, respectively. At the end of study period, mice were sacrificed with the exposure to 
$\mathrm{CO}_{2}$. The heparin mixed samples were centrifuged at $10000 \mathrm{rpm}$ for $10 \mathrm{~min}$ and plasma was stored for further analysis in $-80^{\circ} \mathrm{C} .150 \mu \mathrm{l}$ of plasma and $150 \mu \mathrm{l}$ of ethanol was mixed and vortexed for $1 \mathrm{~h}$. The mixture was centrifuged at $12000 \mathrm{rpm}$ for $15 \mathrm{~min}$ and the supernatant was used to calculate the CDDP concentration. The CDDP was measured by ion coupled plasma mass spectrometry (ICP-MS, PerkinElmer Corporation, USA).

\section{Statistical analysis}

$P$ value $<0.05$ was considered statistically significant. Quantitative data were expressed as mean \pm SD. Statistical comparisons were made by one-way ANOVA analysis and Student's t-test.

\section{Results}

\section{Particle size and surface charge characterization of particles}

The man particle size and surface charge of particles were evaluated by DLS method. The mean particle size of LP-miCDDP was $145.2 \pm 1.25 \mathrm{~nm}$ while the particle size increased to $168.4 \pm 1.34 \mathrm{~nm}$ for CD/LPmiCDDP after the surface conjugation with CD59 antibody. CD/LP-miCDDP exhibited a surface charge of $16.2 \pm 1.61 \mathrm{mV}$ which could help to internalize the particles in the cancer cells (Figure 2a). Several reports have provided evidences that the cellular uptake of nanoparticles is directly related to its size. The nanoparticles of size between $100-200 \mathrm{~nm}$ are reported to preferentially accumulate in the tumor tissues than the normal tissues owing to enhanced permeation and retention (EPR) effect. In this regard, CD/LPmiCDDP showed a smaller particle size with an optimal surface charge sufficient for enhanced accumulation in the tumor tissues.

The surface morphology of the CD/LP-miCDDP was evaluated by TEM (Figure 2b). As shown, particles were perfectly spherical in shape and well-dispersed on the copper grid. The surface modification of particle did not influence the shape of the particles.

\section{CD/LP-miCDDP enhanced cellular accumulation in HeLa cells-CLSM and Flow cytometer analysis}

The target specificity of CD59 antibody conjugated liposome was compared with the non-targeted liposome by means of confocal laser scanning microscopy (CLSM) (Figure 3a). Rhodamine B was loaded in the liposome as a fluorescence tracker and lysosomes were stained with Lysotracker Green and nucleus was stained with DAPI (blue color). The HeLa cells were exposed with CD/LP-miCDDP and LPmiCDDP and incubated for $2 \mathrm{~h}$. As shown, CD/LP-miCDDP exhibited a remarkably higher cellular accumulation in the cancer cells compared to that of LP-miCDDP treated cells. Merged image clearly indicates the presence of liposomes in the lysosome and cytoplasm region and none in the nucleus indicating a typical receptor-mediated cellular uptake. A substantially higher uptake in case of CD/LP- 
miCDDP was believed to be due to the CD59-based receptor uptake. As shown (Figure 3b), CD/LPmiCDDP showed a significantly higher shift in the fluorescence histogram than that of non-targeted liposomes.

\section{MiR-1284 downregulates HMGB1 -Western blot analysis}

Keeping this in mind, miR-1284 effect on the cervical cancer cells were analyzed by Western blotting (Figure 4a). As expected, protein levels of HMGB1 were significantly downregulated in the HeLa cells by miR-1284 mimics while mutant miR did not have any effect on the protein indicating the potential of $\mathrm{miR}-1284$.

\section{Combination of miR-1284 and CDDP in the cell viability}

Here, we have performed a cell viability analysis of effect of miR-1284 mimics a mutant miR on the HeLa cancer cells. As shown (Figure 4b), miR-1284 showed a typical concentration-dependent cell killing effect in the cervical cancer cells whereas mutant miR did not have any effect on the proliferation of cancer cells. Next, we have evaluated the combinational effect of miR-1284 and CDDP in HeLa cancer cells. We have treated the cells were free CDDP, LP-miCDDP and CD/LP-miCDDP and incubated for $24 \mathrm{~h}$ (Figure 4c). As shown, all the formulation exhibited a concentration-dependent cytotoxic effect in the proliferation of cervical cancer cells.

\section{Apoptosis analysis-Hoechst 33342 staining}

The apoptotic effect of each formulation was first determined by Hoechst 33342 staining analysis (Figure 5). The cells were treated with respective formulations and then stained with Hoechst 33342 and observed for changes in cell morphology in the fluorescence microscope. As shown, cell nuclei were perfectly round and lots of cells were attached firmly with the culture plate. However, cells treated with miR-1284 and CDDP resulted in slight apoptosis as observed from the distorted cells and cells undergoing apoptosis. Most remarkable effect was seen in cells treated with CD/LP-miCDDP where remarkable cell/nuclei rupturing and apoptotic bodies were observed. The morphological changes in the cell nuclei of CD/LP-miCDDP treated cells were markedly greater than control cells. Moreover, remarkable decrease in the total cell attached to the plate was observed in CD/LP-miCDDP group indicating the appreciable apoptosis effect of the formulation in the cervical cancer cells.

\section{Apoptosis analysis-Flow cytometer}

The quantitative apoptosis effects of formulations were studied by flow cytometer after staining with Annexin V-FITC and PI (Figure 6). The Annexin V-FITC detects the early apoptosis cells by binding with high affinity to the extracellular phosphatidylserine which is exposed in the cells undergoing apoptosis, 
while, PI penetrates the dead cells through damaged membrane and exhibited red fluorescence. As shown, CD/LP-miCDDP exhibited the maximum apoptosis effect ( 60\%) compared to CDDP ( 20\%) or miR-1284 ( 12\%) treated cells indicating the superior anticancer effect in the cancer cells. LP-miCDDP also exhibited the appreciable apoptosis effect of $\sim 35 \%$ indicating the potential of combinational therapeutics and carrier-based drug delivery.

\section{Pharmacokinetic analysis in animals}

Following the enhanced anticancer effect of formulations, we have evaluated the pharmacokinetic performance of free drug and formulations in SD rats (Figure 7). Animals were divided into 3 groups and formulations were administered. As shown, free CDDP was immediately removed from the blood circulation within $4 \mathrm{~h}$ of administration consistent with many reports regarding the free drug behavior. On the other hand, LP-miCDDP and CD/LP-miCDDP significantly prolonged the blood circulation of encapsulated drug until at least $24 \mathrm{~h}$ after the administration.

\section{Discussion}

Cervical cancer is one of the gynecological cancer responsible most cancer-related deaths in women. Though radiotherapy and surgery are common option in case of advanced cervical cancer, however this standard treatment is unavailable in many developing countries. Based on the guidelines of NCCN for cervical cancer, cisplatin (CDDP) is considered as a first line of treatment option. However, chemotherapy with CDDP results in severe dose-limiting toxicity such as nephrotoxicity or hepatotoxicity and leads to severe drug resistance. Therefore, potential therapeutic approaches by which drug toxicity could be reduced and chemosensitivity of CDDP towards cervical cancer could be increase is perused continuously. In this study, we have selected miR-1284 to increase the chemosensitivity of CDDP for the treatment of cervical cancers. We have prepared unique CD59sp-conjugated CDDP/miR-1284-loaded liposomes for the effective treatment of cervical cancers.

A substantially higher uptake in case of CD/LP-miCDDP was believed to be due to the CD59-based receptor uptake. The CD59 antibody will recognize the corresponding receptor overexpressed in the cancer cells that promote the enhanced internalization of CD/LP-miCDDP in the cancer cells. The quantitative analysis of cellular uptake was performed by flow cytometer. A significant shift in the fluorescence histogram is proof of its enhanced cellular accumulations in the HeLa cells. These results were consistent with the previous reports of higher cellular uptake and higher therapeutic efficacy with CD59-tagreted carrier system in the cancer cells.

Towards the treatment of any cancer, chemotherapy is effective in the beginning however it loses its effectiveness due to the development of chemoresistance over a period of time. CDDP though very potent develops chemoresistance and therefore required to give higher doses which in turn results in severe toxicity. In this regard, it has been reported that miR-1284 could modulate the drug resistance in gastric cancer cells by targeting EIF4 A1. As expected, protein levels of HMGB1 were significantly downregulated 
in the HeLa cells by miR-1284 mimics while mutant miR did not have any effect on the protein indicating the potential of miR-1284. It is worth noting that HNGB1 has been demonstrated to possess oncogenic role in human cancers [22].

Earlier reports have revealed that downregulation of HMGB1 could lead to the inhibition of cell proliferation. Results clearly highlight our expectation that downregulation of HMGB1 could be directly related to the proliferation of cervical cancer cells [23]. It is worth noting that CD59-antibody conjugated formulation exhibited a significantly lower cell viability compared to that of free CDDP. The combination of miR-1284 and CDDP could result in a synergistic anticancer effect on the cervical cancer cells. We expect that miR-1284 might increase the chemosensitivity of HeLa cancer cells and thereby leading to enhanced cell killing effect. It must be noted that the CD/LP-miCDDP exhibited significantly lower cell viability than that of LP-miCDDP attributed to its higher cellular internalized as observed in CLSM and flow cytometer analysis. The IC50 value of CDDP, LP-miCDDP and CD/LP-miCDDP was observed to be $12.4 \mu \mathrm{g} / \mathrm{ml}, 7.23 \mu \mathrm{g} / \mathrm{ml}$ and $3.12 \mu \mathrm{g} / \mathrm{ml}$, respectively, consistent with the cell viability analysis. Blank liposome did not evoke any cytotoxic effect in all tested concentration indicating the biocompatible and safe properties of carrier system. The enhanced anticancer effect of CD/LP-miCDDP was mainly attributed to increased chemosensitivity of CDDP towards the HeLa cells sue to the presence of miR1284 that acted in a synergistic manner. Besides, CD59 was specific towards its receptor overexpressed in the cervical cancer cells that resulted in enhanced accumulation of liposomes and exhibited higher therapeutic effect.

LP-miCDDP and CD/LP-miCDDP significantly prolonged the blood circulation of encapsulated drug until at least $24 \mathrm{~h}$ after the administration. The $\mathrm{AUC}_{(\mathrm{o}-\mathrm{t})}$ of CD/LP-miCDDP showed a 6.9 fold higher value than that of free CDDP. Similarly, CD/LP-miCDDP showed an 8-fold decrease in the clearance (CL) and 3.6-fold higher $t_{1 / 2}$ compared to that of free CDDP. A higher plasma concentration will eventually benefit the anticancer therapy as there will be more chances of tumor accumulation. At the same time, it will minimize the chance of off target to other organs that will substantially reduce the severe adverse effects associated with the CDDP.

\section{Conclusion}

In summary, we successfully designed the CD59sp-conjugated miRNA-1284/CDDP-loaded liposomes for the enhanced therapeutic effect against cervical cancers. A substantially higher uptake in case of CD/LPmiCDDP was believed to be due to the CD59-based receptor-mediated cellular internalization. MiR-1284 showed a typical concentration-dependent cell killing effect in the cervical cancer cells owing to the downregulation of HMGB1. CD59-antibody conjugated formulation exhibited a significantly lower cell viability compared to that of free CDDP and non-targeted LP-miCDDP. The combination of miR-1284 and CDDP could result in a synergistic anticancer effect on the cervical cancer cells. The IC50 value of CDDP, LP-miCDDP and CD/LP-miCDDP was observed to be $12.4 \mu \mathrm{g} / \mathrm{ml}, 7.23 \mu \mathrm{g} / \mathrm{ml}$ and $3.12 \mu \mathrm{g} / \mathrm{ml}$, respectively. CD/LP-miCDDP exhibited remarkable cell/nuclei rupturing and apoptotic bodies' formation indicating a severe apoptosis effect in HeLa cancer cells. Flow cytometer analysis showed that CD/LP-miCDDP 
resulted in maximum apoptosis effect ( 60\%) compared to CDDP ( 20\%) or miR-1284 ( 12\%) treated cells indicating the superior anticancer effect in the cancer cells. Finally, CD/LP-miCDDP significantly prolonged the blood circulation of encapsulated drug in rats with significantly higher $\mathrm{AUC}_{(\mathrm{o-t})}, \mathrm{t}_{1 / 2}$ and lower CL compared to that of free CDDP. Overall, CD/LP-miCDDP could hold a promising potential in the treatment of cervical cancers.

\section{Abbreviations \\ CDDP-Cisplatin}

\section{LP-Liposome}

miR-MiRNA

\section{CD/LP-miCDDP - CD59 antibody-conjugated miRNA-1284/CDDP- loaded liposomes}

\section{LP-miCDDP - miRNA-1284/CDDP-loaded liposomes}

\section{EPR - enhanced permeation and retention}

\section{Declarations}

\section{AUTHOR'S CONTRIBUTION}

LW carried out the main experimental parameters. TTL have carried out the cell-based assays and protocols. TTL has designed and written the entire manuscript. All authors have read and approved the manuscript.

\section{FUNDINGS}

The funding grant was secured by Ting-Ting Liang from Weifang No.2 People's Hospital, Weifang. The funding was used to procure all the materials and animals for the study.

\section{ETHICS APPROVAL AND CONSENT TO PARTICIPATE:}

Not applicable 


\section{ACKNOWLEDGEMENT:}

Not applicable

\section{CONSENT FOR PUBLICATION:}

Not applicable

\section{AVAILABILITY OF DATA AND MATERIALS:}

Not applicable

\section{DECLARATIONS}

The authors report no conflict of interest.

\section{References}

1. Kanavos P. The rising burden of cancer in the developing world. Ann Oncol. 2006; 17: viii15-viii23.

2. Jin T, Wu X, Yang H, Liu M, He Y, He X, Shi X, Wang F, Du S, Ma Y, Bao S, Yuan D. Association of the miR-17-5p variants with susceptibility to cervical cancer in a Chinese population. Oncotarget 2016;7:76647-76655.

3. Siegel R, Ma J, Zou Z, Jemal A. Cancer statistics, 2014. CA Cancer J Clin 2014;64:9-29

4. Hirte $H$, Kennedy EB, Elit L, Fung Kee Fung M. Systemic therapy for recurrent, persistent, or metastatic cervical cancer: a clinical practice guideline. Curr Oncol. 2015;22:211-219.

5. Scatchard K, Forrest JL, Flubacher M, Cornes P, Williams C. Chemotherapy for metastatic and recurrent cervical cancer. Cochrane Database Syst Rev. 2012;10:CD006469.

6. Wang Z, Lv J, Zhang T. Combination of IL-24 and cisplatin inhibits angiogenesis and lymphangiogenesis of cervical cancer xenografts in a nude mouse model by inhibiting VEGF, VEGF-C and PDGF-B. Oncol Rep. 2015;33:2468-2476.

7. Shin VY, Chu KM. MiRNA as potential biomarkers and therapeutic targets for gastric cancer. World J Gastroenterol. 2014;20:10432-10439.

8. Ruan K, Fang X, Ouyang G. MicroRNAs: novel regulators in the hallmarks of human cancer. Cancer Lett. 2009;285:116-126

9. Li J, Jin H, Yu H, Wang B, Tang J. miRNA1284 inhibits cell growth and induces apoptosis of lung cancer cells. Mol Med Rep. 2017;16:3049-3054.

10. Huang M, Wu L, Luo S, Qin H, Yang Y, Chen J, Li Z, Qin Y. MicroRNA-1284 inhibits proliferation and induces apoptosis in SGC-7901 human gastric cancer cells. Biotechnol Lett. 2017;39:33-38. 
11. Pan C, Wang D, Zhang Y, Yu W. MicroRNA-1284 inhibits cell viability and induces apoptosis of ovarian Cancer cell line OVCAR3. Oncol Res. 2016;24:429-435.

12. Oberoi HS, Nukolova NV, Kabanov AV, Bronich TK. Nanocarriers for delivery of platinum anticancer drugs. Adv Drug Deliv Rev. 2013;665:1667-1685.

13. Panyam J, Labhasetwar V. Biodegradable nanoparticles for drug and gene delivery to cells and tissue. Adv Drug Deliv Rev. 2003;55:329-347

14. Lockhart JN, Stevens DM, Beezer DB, Kravitz A, Harth E. Dual drug delivery of tamoxifen and quercetin: regulated metabolism for anticancer treatment with nanosponges. $\mathrm{J}$ Control Release. 2015;220(Pt B): 751-757.

15. Sagnella SM, Gong X, Moghaddam MJ, et al. Nanostructured nanoparticles of self-assembled lipid pro-drugs as a route to improved chemotherapeutic agents. Nanoscale. 2011;3(3):919-924.

16. Ichihara $\mathrm{H}$, Motomura M, Matsumoto $\mathrm{Y}$. Therapeutic effects and anti-metastasis effects of cationic liposomes against pancreatic cancermetastasis in vitro and in vivo. Biochem Biophys Res Commun. 2019, doi: 10.1016/j.bbrc.2019.02.116.

17. Zhang X, Dai F, Chen J, Xie X, Xu H, Bai C, Qiao W, Shen W. Antitumor effect of curcumin liposome after transcatheter arterial embolization in VX2 rabbits. Cancer Biol Ther. 2019, doi: 10.1080/15384047.2018.1550567

18. Day KC, Lorenzatti Hiles G, Kozminsky M, Dawsey SJ, Paul A, Broses LJ, et al. HER2 and EGFR Overexpression Support Metastatic Progression of Prostate Cancer to Bone. Cancer Research. 2017;77:74-85

19. Gasparetto M, Smith CA. ALDHs in normal and malignant hematopoietic cells: Potential new avenues for treatment of AML and other blood cancers. Chemico-Biological Interactions. 2017;276:46-51.

20. Afshar-Kharghan V. The role of the complement system in cancer. The Journal of Clinical Investigation 2017;127:780-9.

21. Li B, Gao MH, Chu XM, Xu YJ, Yang F. Identification of a novel short peptide seal specific to CD59 and its effect on HeLa cell growth and apoptosis. Cellular Oncology 2012;35:355-65

22. Cao W, Wei W, Zhan Z, Xie Y, Xiao Q. MiR-1284 modulates multidrug resistance of gastric cancer cells by targeting EIF4A1. Oncol Rep. 2016;35:2583-2591

23. Wu L, Yang $L$. The function and mechanism of HMGB1 in lung cancer and its potential therapeutic implications. Oncol Lett. 2018;15:6799-6805.

\section{Figures}




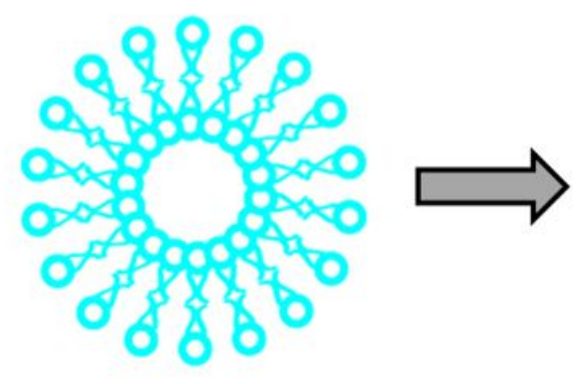

Blank liposome

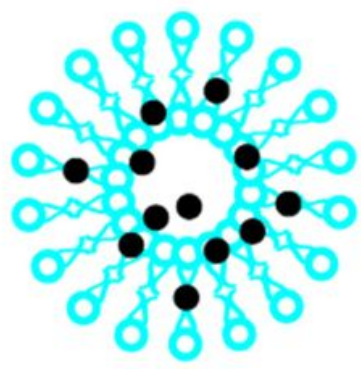

CDDP-loaded liposome

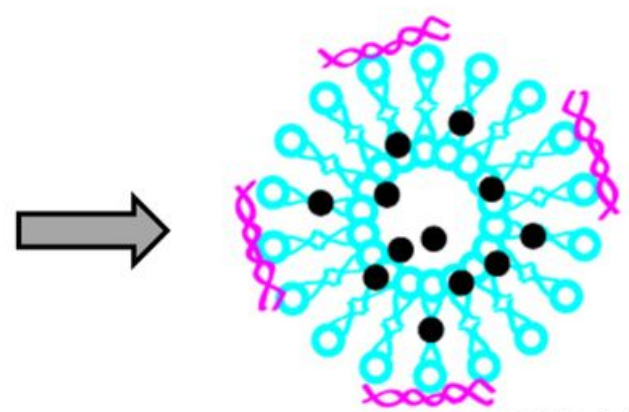

miRNA/CDDP-

loaded liposome

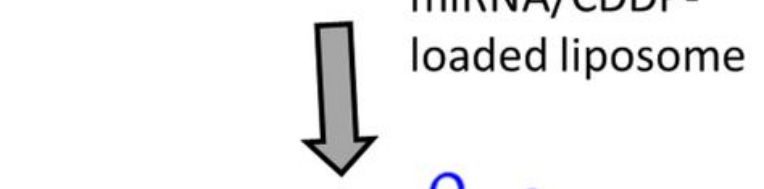

\section{Figure 1}

Graphical presentation of preparation of CD59sp-conjugated CDDP/miRNA-1284 liposome. The CDDP was loaded in the liposome shell and miRNA was loaded on the surface of liposomes.

(a)

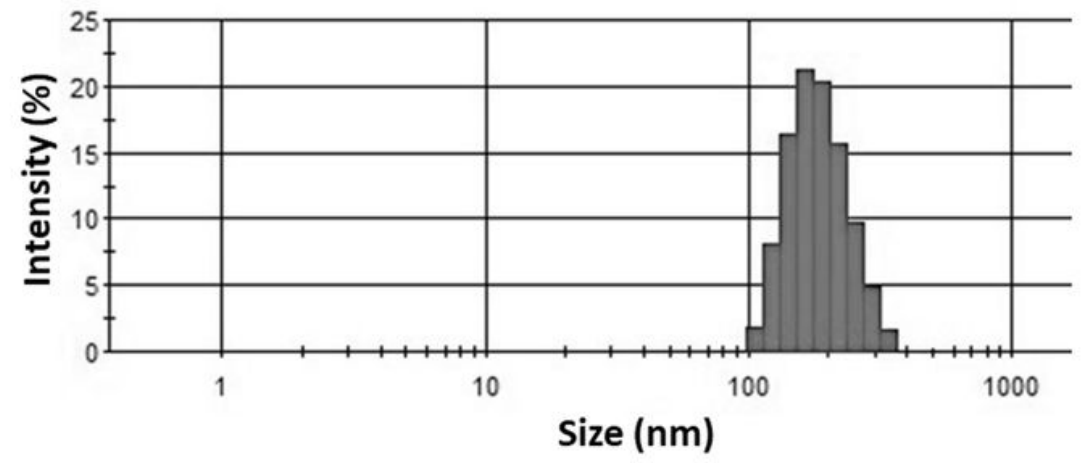

(b)

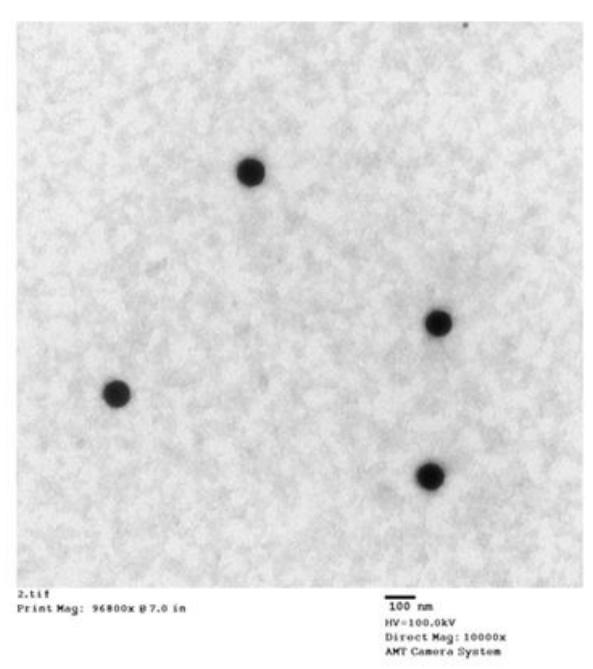


Figure 2

(a) Physicochemical characterization of CD/LP-miCDDP, particle size distribution using dynamic light scattering (DLS); (b) morphology analysis of CD/LP-miCDDP using transmission electron microscopy (TEM)
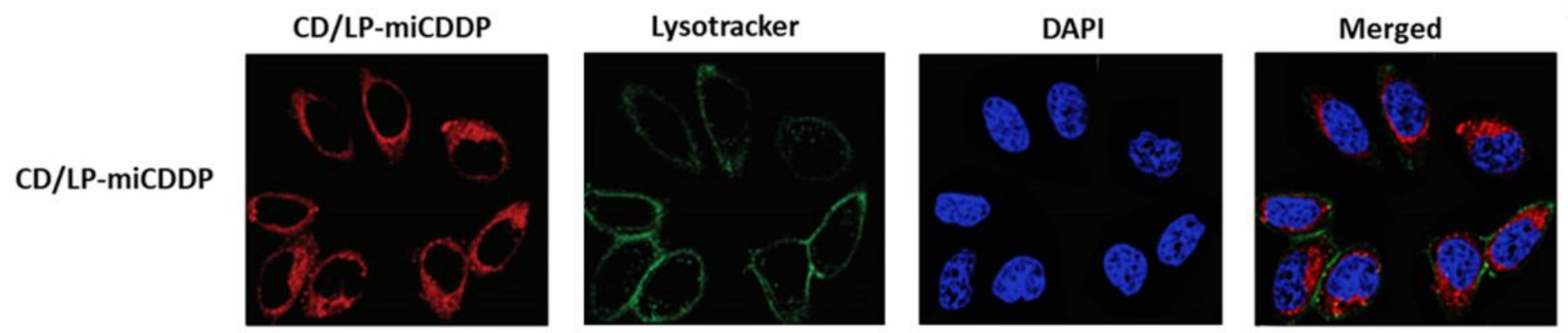

(a)
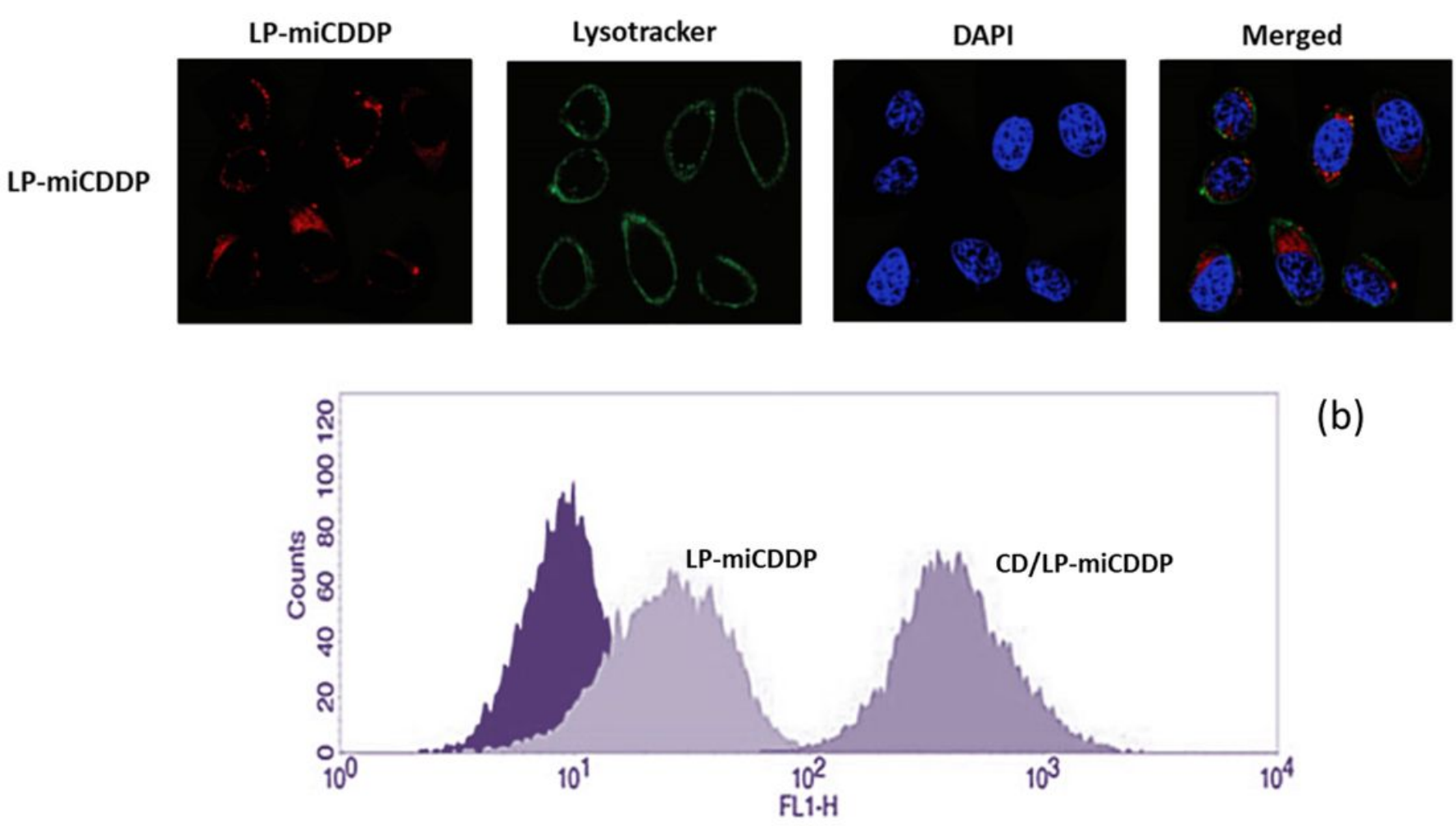

(b)

\section{Figure 3}

(a) Cellular uptake analysis of LP-miCDDP and CD/LP-miCDDP in HeLa cells using confocal laser scanning microscopy (CLSM). The Rhodamine B was used as a fluorescent tracker and the cells were stained with Lysotracker Green and DAPI as a respective lysosome and nucleus tracker; (b) flow cytometer analysis of cellular uptake after incubation of liposomes to cells for $2 \mathrm{~h}$ 
(a)

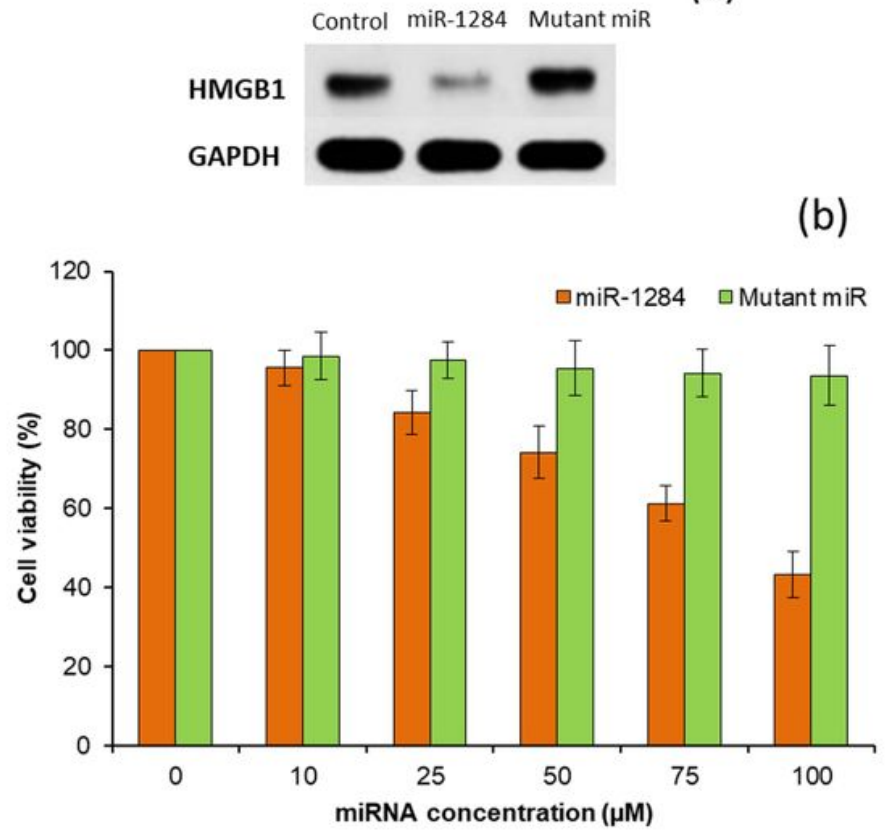

(c)

(b)

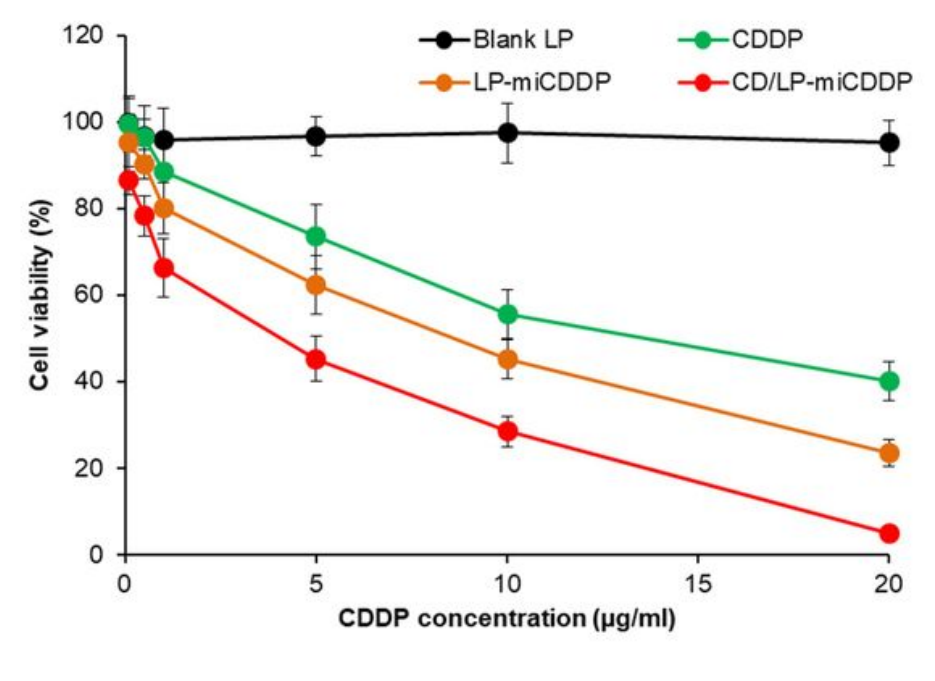

Figure 4

(a) Western blot analysis of protein expression of HMGB1 in HeLa cancer cells; (b) cell viability analysis of miR-1284 and Mutant miR on the HeLa cancer cells in a dose-dependent manner; (c) cell viability analysis of free CDDP and formulations on the viability of cancer cells. The cell viability was determined by MTT assay. 
Control

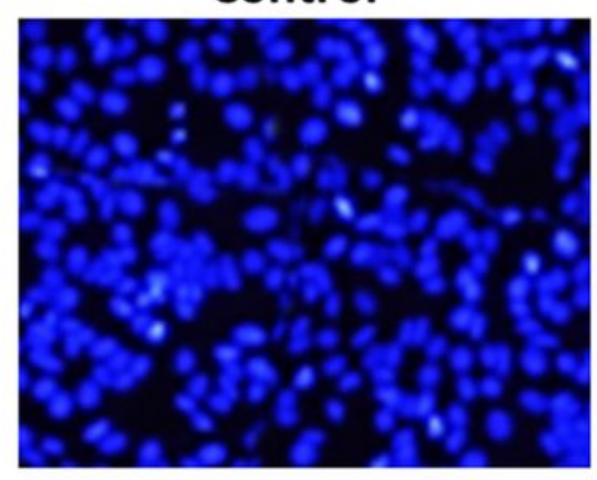

miRNA-1284

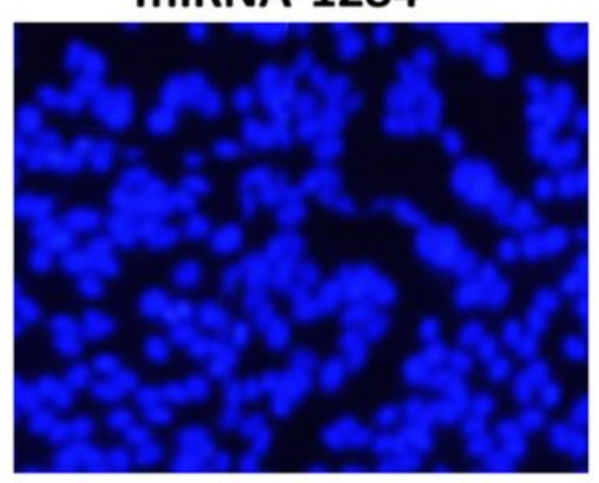

CDDP

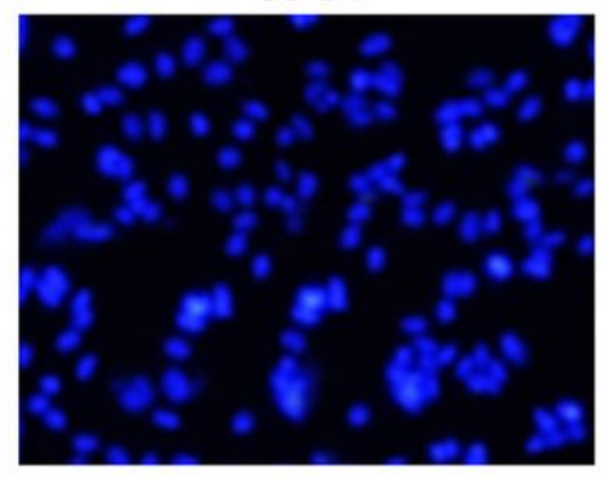

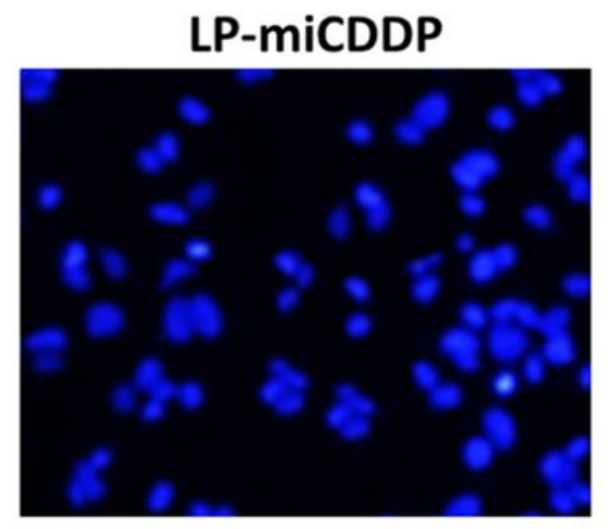

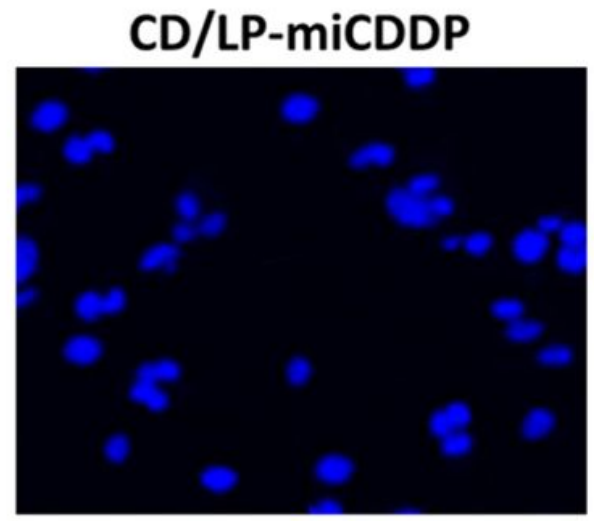

Figure 5

Apoptosis analysis of HeLa cancer cells after treatment with miR-1284, CDDP, LP-miCDDP and CD/LPmiCDDP using Hoechst 33342 staining. 

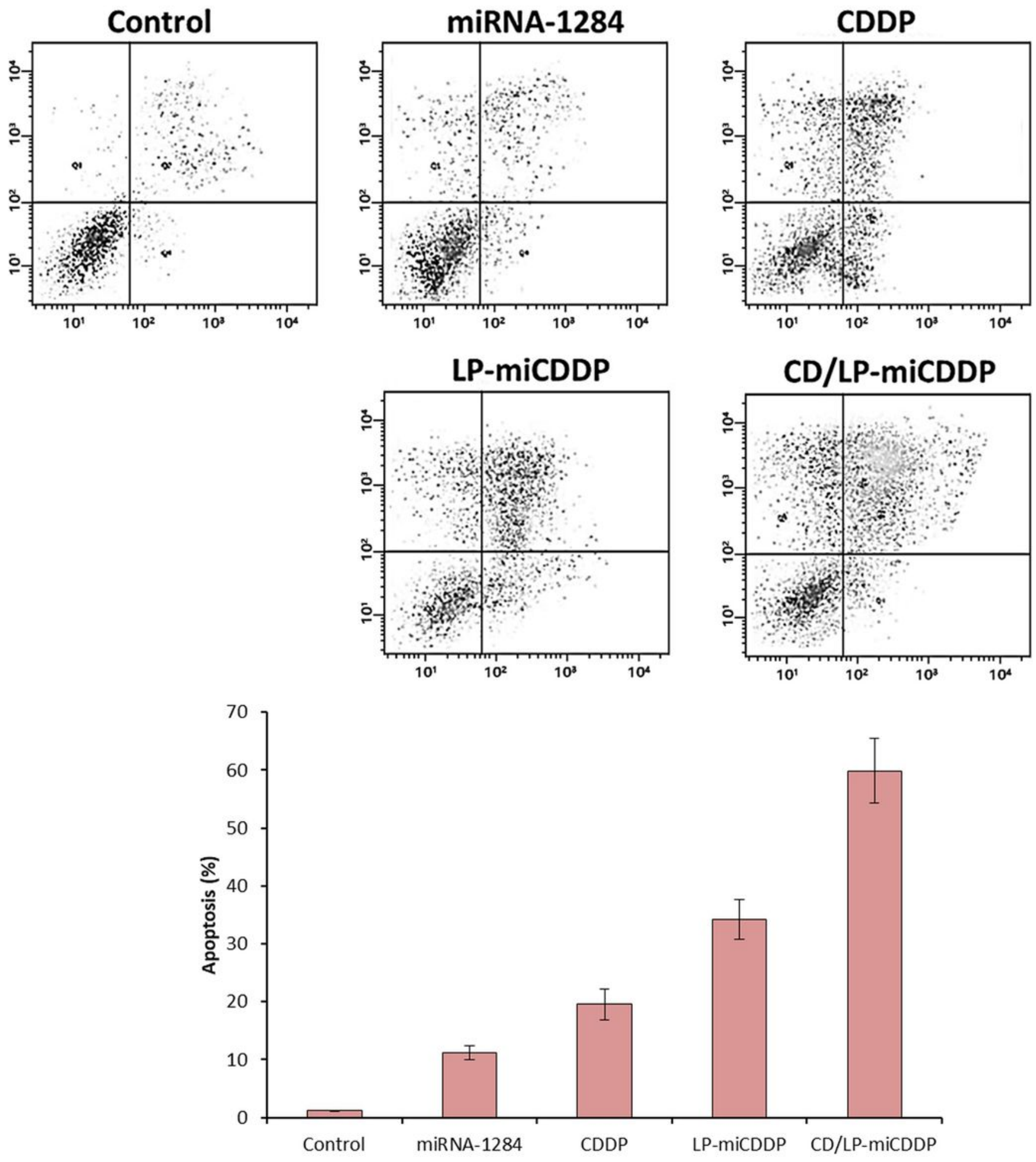

Figure 6

Quantitative apoptosis analysis of of HeLa cancer cells after treatment with miR-1284, CDDP, LP-miCDDP and CD/LP-miCDDP. The cells were stained with Annexin V-FITC and PI and 10000 cell events were observed at flow cytometer 


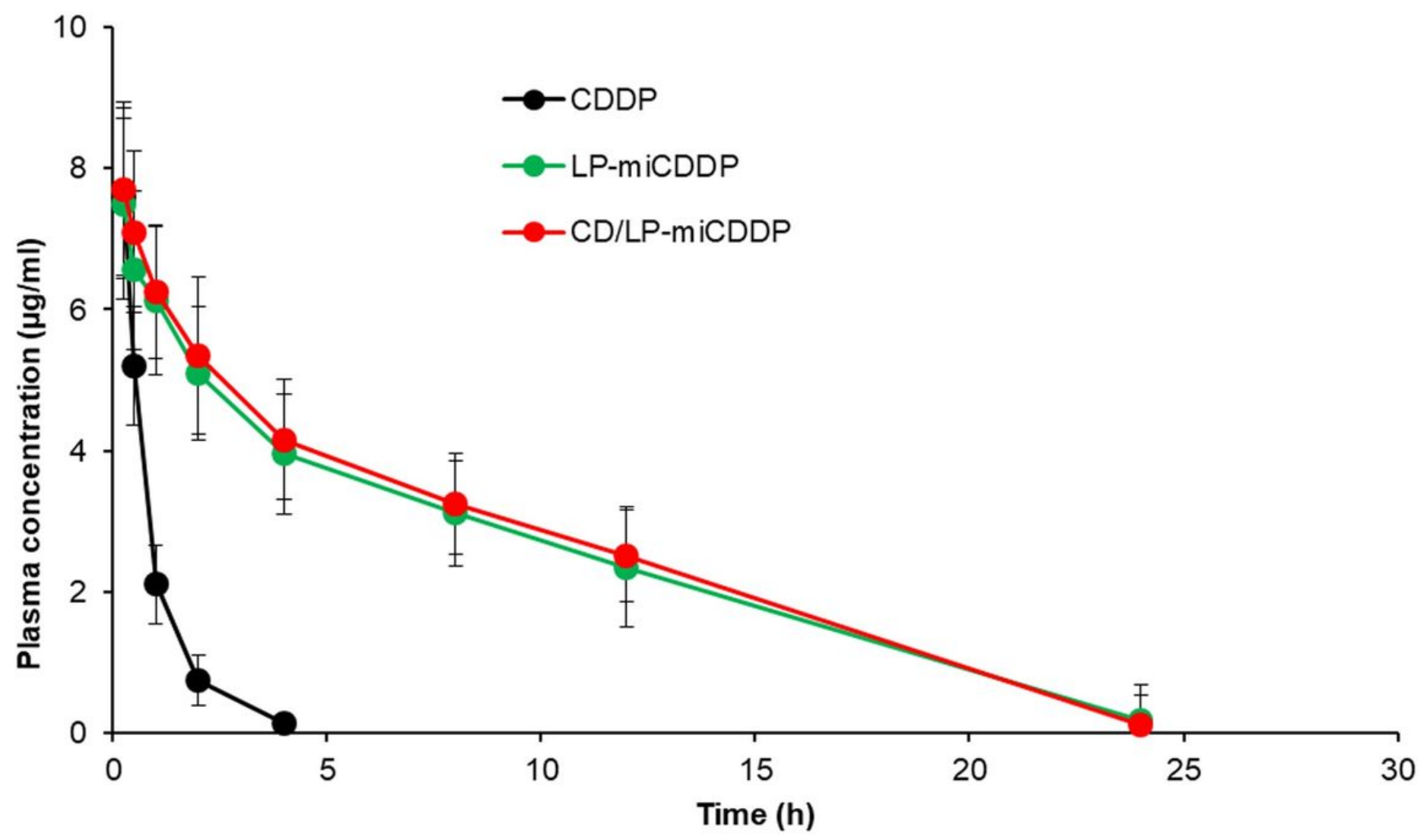

Figure 7

Plasma concentration-time profile of free CDDP, LP-miCDDP and CD/LP-miCDDP. The free drug and formulations were administered in SD rats by tail-vein injection and studied until $24 \mathrm{~h}$.

\section{Supplementary Files}

This is a list of supplementary files associated with this preprint. Click to download.

- ARRIVE.pdf 